\title{
Erratum to: Ethnic/Racial Disparities in the Fetal Growth Outcomes of Ecuadorian Newborns
}

\author{
M. Margaret Weigel ${ }^{1,2,3} \cdot$ Maria Elena Caiza Sanchez ${ }^{4}$
}

Published online: 2 August 2016

(c) Springer Science+Business Media New York 2016

Erratum to: J Immigrant Minority Health (2013) 15:198-206

DOI 10.1007/s10903-011-9571-5

The corresponding author's name should read as M. Margaret Weigel instead of Margaret Weigel $M$. where the given name is M. Margaret and the family name is Weigel.

This has been corrected with this erratum.

The online version of the original article can be found under doi:10.1007/s10903-011-9571-5.

M. Margaret Weigel

mmweigel@utep.edu

1 Department of Public Health Sciences, College of Health Sciences, University of Texas at El Paso, $500 \mathrm{~W}$. University Ave, El Paso, TX 79968, USA

2 Center for Interdisciplinary Health Research and Evaluation, College of Health Sciences, University of Texas at El Paso, El Paso, TX, USA

3 Human Immunology and Nutrition Research Laboratory College of Health Sciences, University of Texas at El Paso, El Paso, TX, USA

4 Ministerio de Salud Publica, Quito, Ecuador 